\title{
Advances in Cryo-EM structure-based methods in membrane protein drug discovery
}

Michael Hennig

leadXpro AG, United States

Integral membrane proteins are drug targets for the majority of all approved medicines. While structure-based drug discovery on soluble proteins is managed well within the project timelines and portfolio changes in pharmaceutical industry, transmembrane proteins are still underexplored because of their challenges to be expressed, purified and made them work for drug ligand interaction analysis by biophysical methods and high resolution structure determination.

The presentation includes recent technology advances and their application to relevant drug targets. Examples will include the cryo-EM structure of the human TRPV4 ion-channel [1] with bound small molecule agonist. The agonist binding activates the channel opening with a significant conformational change enabling direct observation of agonist pharmacology.

LPTDE is a clinically validated antibiotics drug target [2]. Due to its small size of $120 \mathrm{kDa}$ and the monomeric b-sheet transmembrane architecture, high resolution EM analysis turned out to be challenging. Addition of two Pro-Macrobodies (PMb's), which are VHH antibody derived and conformationally rigidified fusion proteins, was essential to enable an EM structure at $2.9 \AA$ resolution. PMb's represent a novel and instrumental tool to enable high resolution EM structure determination on small proteins.

Furthermore, the $2.7 \AA$ EM structure determination of a novel human potassium ion-channel [3] with bound ligand will be presented explaining the mode of channel opening and gives insight of the mechanism of action of disease mutants.

The outlook includes expected further advances in cryo-EM and the observation of structural dynamic of caused by ligand binding. Such insights will further enhance the impact of structures to the better design of compounds as well as enable successful drug discovery on challenging membrane protein targets such as ionchannels at all.

\section{References}

[1] Mathieu Botte, Alexander K. C. Ulrich, Ricardo Adaixo, David Gnutt, Andreas Brockmann, Denis Bucher, Mohamed Chami, Nicolas Bocquet, Ulrich Ebbinghaus-Kintscher, Vera Puetter, Andreas Becker, Ursula Egner, Henning Stahlberg, Michael Hennig and Simon J. Holton, Cryo-EM structural studies of the agonist complexed human TRPV4 ion-channel reveals novel structural rearrangements resulting in an openconformation (2020), https://doi.org/10.1101/2020.10.13.334797

[2] Mathieu Botte, Dongchun Ni, Stephan Schenck1, Iwan Zimmermann3, Mohamed Chami2, Nicolas Bocquet1, Pascal Egloff3, Denis Bucher, Matilde Trabuco, Robert K.Y. Cheng, Janine Brunner, Markus A. Seeger, Henning Stahlberg and Michael Hennig Insight into the lipopolysaccharide translocation by cryo-EM structures of a LptDE transporter in complex with Pro-Macrobodies. Manuscript submitted (2021)

[3] Mathieu Botte, Sophie Huber, Denis Bucher, David Rodriges, Lena Tagmose, Mohamed Chami, Nicolas Bocquet, Michael Hennig and Wassim Abdul Rahman. Manuscript in preparation (2021). 